\title{
Mueller matrix ellipsometry of artificial non-periodic line edge roughness in presence of finite numerical aperture
}

\author{
Martin Foldyna*a, Thomas A. Germer ${ }^{\mathrm{b}}$, Brent C. Bergner ${ }^{\mathrm{c}}$ \\ a'LPICM, Ecole Polytechnique, CNRS, 91128 Palaiseau, France \\ *martin.foldyna@polytechnique.fr; \\ bptical Technology Division, National Institute of Standards and Technology, \\ Gaithersburg, MD 20899, USA \\ 'Spectrum Scientific, Inc., 16692 Hale Avenue, Irvine, CA 92606, USA
}

\begin{abstract}
We used azimuthally-resolved spectroscopic Mueller matrix ellipsometry to study a periodic silicon line structure with and without artificially-generated line edge roughness (LER). The unperturbed, reference grating profile was determined from multiple azimuthal configurations using a generalized ellipsometer, focusing the incident beam into a $60 \mu \mathrm{m}$ spot. We used rigorous numerical modeling, taking into account the finite numerical aperture, introducing significant depolarization effects, and determining the profile shape using a four trapezoid model for the line profile. Data obtained from the artificially perturbed grating were then fit using the same model, and the resulting root-mean-square error (RMSE) values for both targets were compared. The comparison shows an increase in RMSE values for the perturbed grating that can be attributed to the effects of LER.
\end{abstract}

Keywords: Finite numerical aperture, line edge roughness, Mueller matrix ellipsometry, periodic grating

\section{INTRODUCTION}

Line edge roughness (LER) represents one of the challenges of current semiconductor technology process control [1]. The importance of LER increases with an increase of the ratio between LER and target line width, which is becoming one of the largest part of the uncertainty budget of current and future technologies. Optical metrology tools make up an important part of the suite of non-destructive inline process control tools that provide real-time assessment of the quality of production wafers after each lithographic step. There is constant need to assess the effectiveness of optical characterization methods, as new challenges rise from technological progress. One of the challenges is a requirement that measurements be made inside very small (usually smaller than $100 \mu \mathrm{m}$ ) targets. Measurements using spectroscopic ellipsometer techniques require significant focusing of the light beam, with numerical apertures on the order of a few degrees. The numerical aperture of the incident light beam puts additional demands on the optical modeling as well. Furthermore, it requires reevaluation of the sensitivity of the optical methods to the LER. The usual effect of the numerical aperture includes incident angle averaging, which can wash out important spectral features [2] and may decrease the sensitivity to imperfections such as LER.

Previous theoretical studies of the sensitivity of angularly-resolved ellipsometry to LER were performed on large sets of 2D gratings with randomly generated LER. It has been shown that randomly generated LER can be used as a heuristic tool to evaluate its impact on angle-resolved ellipsometric data [3, 4]. Nevertheless, case studies with real samples and measurements are needed in order to confirm the theoretical results. We have prepared periodically-perturbed line gratings and studied the impact of line-width roughness (LWR) on root-mean-square errors (fit residuals) for generalized spectroscopic ellipsometry data [5]. We have shown that the differences are solely due to the LWR using rigorous 2D numerical modeling, which represented the designed artificial LWR. For the purpose of this work we have manufactured samples with pseudo-random LER and applied previously developed optical models to the spectrally-resolved Mueller matrix data. The description of the samples can be found in Section 2, with corresponding scanning electron microscopy images of lines inside the studied targets. In the same section, we provide experimental details and optical methods used to characterize the targets. In Section 3, we provide a detailed description of the rigorous modeling and fitting parameters. Section 4 summarizes and discusses the major results of our work. Finally, we make some conclusions in Section 5. 


\section{EXPERIMENTAL DETAILS AND THEORETICAL METHOD}

The sample studied in this work was manufactured at the University of North Carolina, Charlotte using electron beam lithography followed by etching to make $736 \mathrm{~nm}$ pitch line gratings into a silicon wafer. The nominal height of the lines was $400 \mathrm{~nm}$, and the nominal width of the lines was $300 \mathrm{~nm}$. A scanning electron microscope image taken from the top of the reference target is shown on the left in Fig. 1. Artificially designed LER roughness is represented by non-periodic modulation of the line-width with the nominal depth being $2 \%$ of the line-width (see Fig. 1, right).

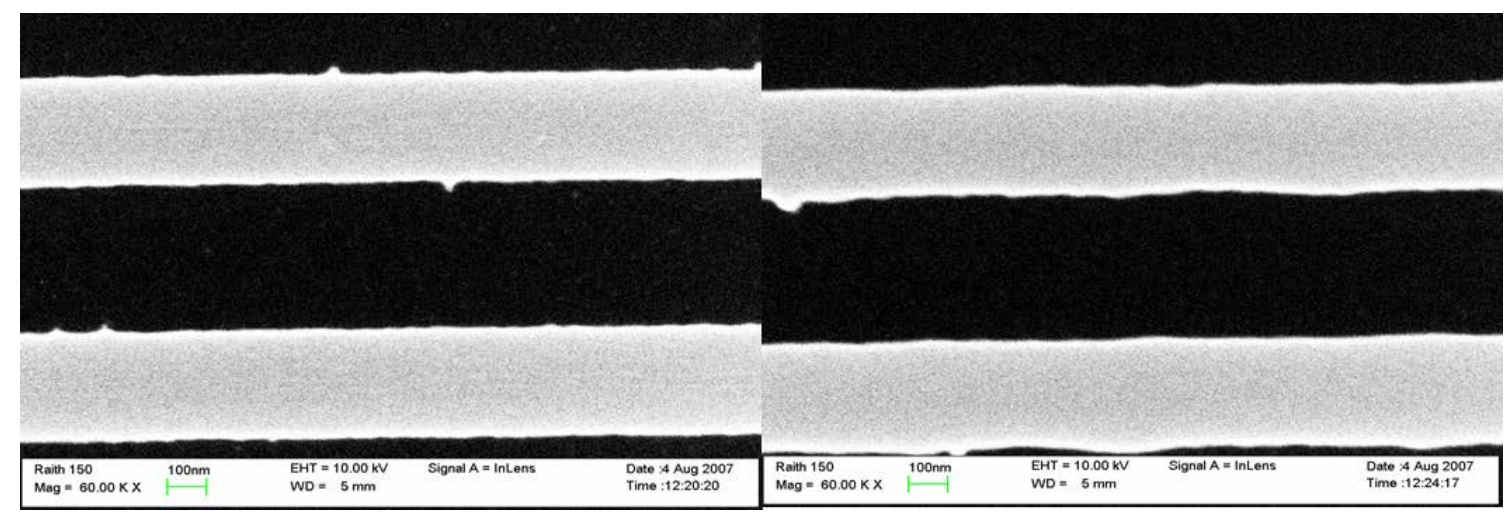

Figure 1. Unperturbed reference grating (left) and grating with artificially designed non-periodic LER (right).

Both samples were measured using a generalized spectroscopic ellipsometer with an automated sample rotation stage, providing 11 elements of the normalized Mueller matrix (elements in the last line of Mueller matrix are not available) at different azimuthal configurations. The instrument uses focusing optics in order to project the beam onto the sample with spot size smaller than $60 \mu \mathrm{m}$ at a fixed incident angle of $65^{\circ}$. Experimental azimuthal angles were chosen from $-180^{\circ}$ to $180^{\circ}$ in increments of $10^{\circ}\left(0^{\circ}\right.$ corresponding to the grating direction). Azimuths of opposite sign and $180^{\circ}$ rotation were simultaneously used during the modeling to decrease the importance of systematic experimental errors, which do not have the same symmetries as the natural physical symmetries present in the optical response of the line grating. It also effectively compensates for the missing off-diagonal elements of the Mueller matrices, considering relations between transposed elements taken at opposite azimuthal angles. The information in off-diagonal elements of the Mueller matrix is important for removing correlations between some profile parameters during the fitting process and for the sensitive determination of precise values of azimuthal offset. It also helps with independent assessment of the grating profile symmetry, as this can be determined directly from appropriate Mueller matrix elements.

Measurements of the unperturbed and perturbed gratings were taken using the same azimuthal angles in order to directly compare the data. The two gratings were also designed with the same mean line width. This helps when comparing raw measured data without the aid of any model, due to the fact that the curves are very similar except in a few spectral regions. Differences between the data taken on the two gratings demonstrate sensitivity of spectroscopic ellipsometry to the magnitude of the periodic line width perturbation.

We would like to confirm that the differences between data are solely due to the line edge perturbations and not some other imperfection in the model or other physical differences between them. In order to do that, we are going to establish a reasonably accurate optical model of the unperturbed grating and explain the remaining differences between the data measured on the perturbed sample. In previous work, we used periodically perturbed targets, for which we could apply biperiodic rigorous coupled-wave analysis (RCWA) to the periodically perturbed grating lines, in order to positively confirm differences in data [5]. In this study, we find the grating profile, which provides sufficiently stable and accurate description of the grating for all possible azimuthal angles, and then to compare the best fits of the unperturbed grating with those of the perturbed one. The differences in the root-mean-square error (RMSE) must be small compared with the RMSE value of the unperturbed lines, in order to exclude possible differences due to the grating profile simplification. Rigorous two dimensional model of the periodically perturbed lines provided explanation for the increase of the RMSE and positively confirmed origin of the differences in the data. From the increase of the RMSE of the perturbed grating, as compared to the small RMSE of the unperturbed one, we can deduce the sensitivity of the method to artificial nonperiodic roughness. 


\section{RIGOROUS THEORY AND STATISTICAL ANALYSIS}

\subsection{Rigorous coupled-wave analysis and Mueller matrix formalism}

Periodic gratings can be modeled using rigorous coupled-wave analysis (RCWA). The method uses Fourier series expansions of the electric and magnetic fields inside the structure in order to express and calculate propagating and evanescent waves in different layers of the structure. Afterwards, tangential field components are matched at the boundaries between the layers, resulting in the determination of the complex reflection and transmission coefficients for each of the diffracted orders. Standard RCWA implementations are based on the original work of Moharam and Gaylord $[6,7]$ using a staircase approximation of the grating profile. The convergence rate is significantly increased for lamellar gratings (especially in the case of absorbing materials) using inverse rules which appeared at first in the work of Lalanne and Morris [8] and were then mathematically proven by Li [9]. The inverse rule approach uses a more consistent treatment of the boundary conditions inside the lamellae, leading to better expansion of the permittivity into Fourier series and, consequently, to better convergence of the numerical algorithm. Another improvement to the original formulation uses the scattering matrix algorithm instead of the original transmittance matrix approach [10]. The latter permits calculations on deeper gratings using more terms in the Fourier series expansions, which would otherwise suffer from finite numerical precision. In the scattering matrix algorithm the modes outside of the structure are organized into two subsets: modes approaching the structure and modes leaving the structure. These improvements allow for more consistent calculation of the modes propagating through the structure and lead to an increased stability of the numerical implementation. Together with the original work of Moharam and Gaylord (or work of Rokushima for anisotropic gratings [11]), these methods are the workhorse for calculating optical response of periodic gratings.

Using the RCWA calculation, the complete complex Jones matrix [12] for every reflected or transmitted diffraction order is determined. The complex Jones matrix $J$ can be defined as

$$
J=\left[\begin{array}{ll}
J_{11} & J_{12} \\
J_{21} & J_{22}
\end{array}\right]=\left[\begin{array}{ll}
J_{p p} & J_{p s} \\
J_{s p} & J_{s s}
\end{array}\right],
$$

where $s$ and $p$ denote the transversal electric (TE) and transversal magnetic (TM) polarizations, respectively. The optical response of the periodic grating to a plane wave can be completely described using the Jones matrix formalism. In some measurements, depolarization can appear as a direct consequence of some experimental imperfections and has to be treated properly. The major source of depolarization considered in this work is the high numerical aperture (NA $\approx 0.065$ ) of the incident beam, which is focused into the very small spot on the sample (less than $60 \mu \mathrm{m}$ in diameter). As a result, experimental data show depolarization due to the incoherent superposition of the optical response over all incidence and azimuthal angles from the experimental aperture range.

In the most general case, the linear optical response can be described by the Mueller matrix formalism, which includes effects of depolarization on the measured signal. Mueller matrices are described using $4 \times 4$ real matrices of the form:

$$
M=\left[\begin{array}{llll}
M_{11} & M_{12} & M_{13} & M_{14} \\
M_{21} & M_{22} & M_{23} & M_{24} \\
M_{31} & M_{32} & M_{33} & M_{34} \\
M_{41} & M_{42} & M_{43} & M_{44}
\end{array}\right] .
$$

In order to model depolarizing Mueller matrices, the incoherent superposition of Mueller matrices of the representative set of incident directions for the given numerical aperture needs to calculated. Weights of selected points can be acquired using the known value of the numerical aperture [2], and the resulting depolarizing Mueller matrix can be written as

$$
M_{i j}^{d}=\frac{1}{\sum_{k} w_{k}} \sum_{k} w_{k} M_{i j, k},
$$

where $k$ denotes the selected direction index, $i$ and $j$ are the Mueller matrix coefficients and $w_{k}$ denotes the appropriate weight. Modeling of the depolarizing experimental data has substantial impact on the calculation time due to the necessity to calculate multiple incidence configurations. In our numerical implementation, we use nine points 
representing given numerical aperture, which provides sufficient precision for the comparison with the experimental data, but it also increases calculation times by a factor of nine. The most significant impact of the numerical aperture (NA) on the measured data can be seen in spectral regions with sharp spectral features [13].

\subsection{Determining grating profile}

The profile of the grating was determined for each azimuth separately by a least-squares optimization procedure, searching for optimal values of model parameters, which provide the best correspondence between experimental and modeled Mueller matrix data. Searching for the optimal values of parameters (values corresponding to local minimum of the RMSE parametric function) is initiated using a numerical-gradient-based method, and the result is used as an initial guess for a subsequent search using the Levenberg-Marquardt method. The merit function, defined as a function of free parameter set $p$, can be written using all experimentally available elements of normalized Mueller matrices as follows:

$$
\operatorname{RMSE}(p)=\left\{\left.\frac{1}{11 N_{s}-n-1} \sum_{k=1}^{N_{s}} \sum_{i=1}^{3} \sum_{j=1}^{4}\left[M_{i j, k}^{m}(p)-M_{i j, k}^{e}(p)\right]^{2}\right|_{i \neq 1 \vee j \neq 1}\right\}^{1 / 2},
$$

where $M_{i j, k}$ denote the element $M_{i j}$ of the $k$-th spectral Mueller matrix, which is marked by $m$ for the modeled and $e$ for the experimental data, respectively. Numbers $N_{s}$ and $n$ denote the total number of spectral points and the number of free model parameters, respectively.

In our fits, we modeled the grating profile with four trapezoids, illustrated in Fig. 2. The overall number of free parameters was 11: the total height of the line, heights of top, third, and bottom trapezoids; four trapezoid base widths; top width; offset to the nominal azimuthal angle; and the thickness of the silicon dioxide layer on the top. Different azimuthal angles were fitted separately in order to provide more reliable values of model parameters as the correlations between parameters change for different azimuthal angles. We combined data from opposite azimuthal angles, which do not provide new physical information (modeled values for azimuthal angle $\varphi$ can be used to directly calculate values for azimuth $-\varphi$ ), and $180^{\circ}$ symmetric azimuths in order to decrease impact of systematic experimental errors and partially compensate for the missing four Mueller matrix elements (the last line of the matrix).

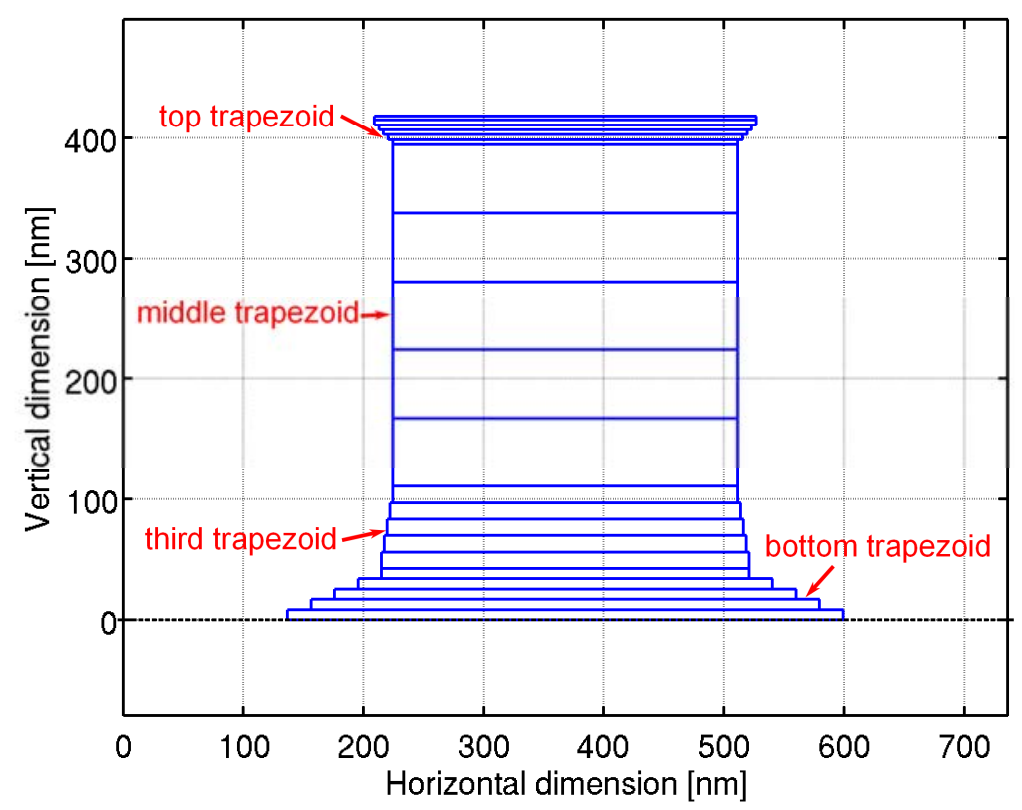

Figure 2. Fitted grating profile represented by layers as used directly in the RCWA code, determined for an azimuth of $30^{\circ}$. The profile is composed of four trapezoids on top of each other with a clear presence of an overhang on the top of the grating. 


\section{RESULTS AND DISCUSSIONS}

Normalized, spectrally-resolved Mueller matrix data measured at the planar configuration inside the reference and the artificial LER targets is shown in Fig. 3. All block off-diagonal elements of the Mueller matrix are zero in this case and the element $M_{22}$ should have the constant value of 1 . We can see that there are horizontal shifts between both sets of data, which can be attributed to the fact that the reference grating does not necessarily have exactly the same line width as an effective average line-width of the perturbed grating. This validates our approach to not compare the raw data directly, but to compare results of the grating profile fits for both targets. Except the horizontal shift, we can also observe some other differences between the curve shapes which are likely to be attributed to the effects of LER.
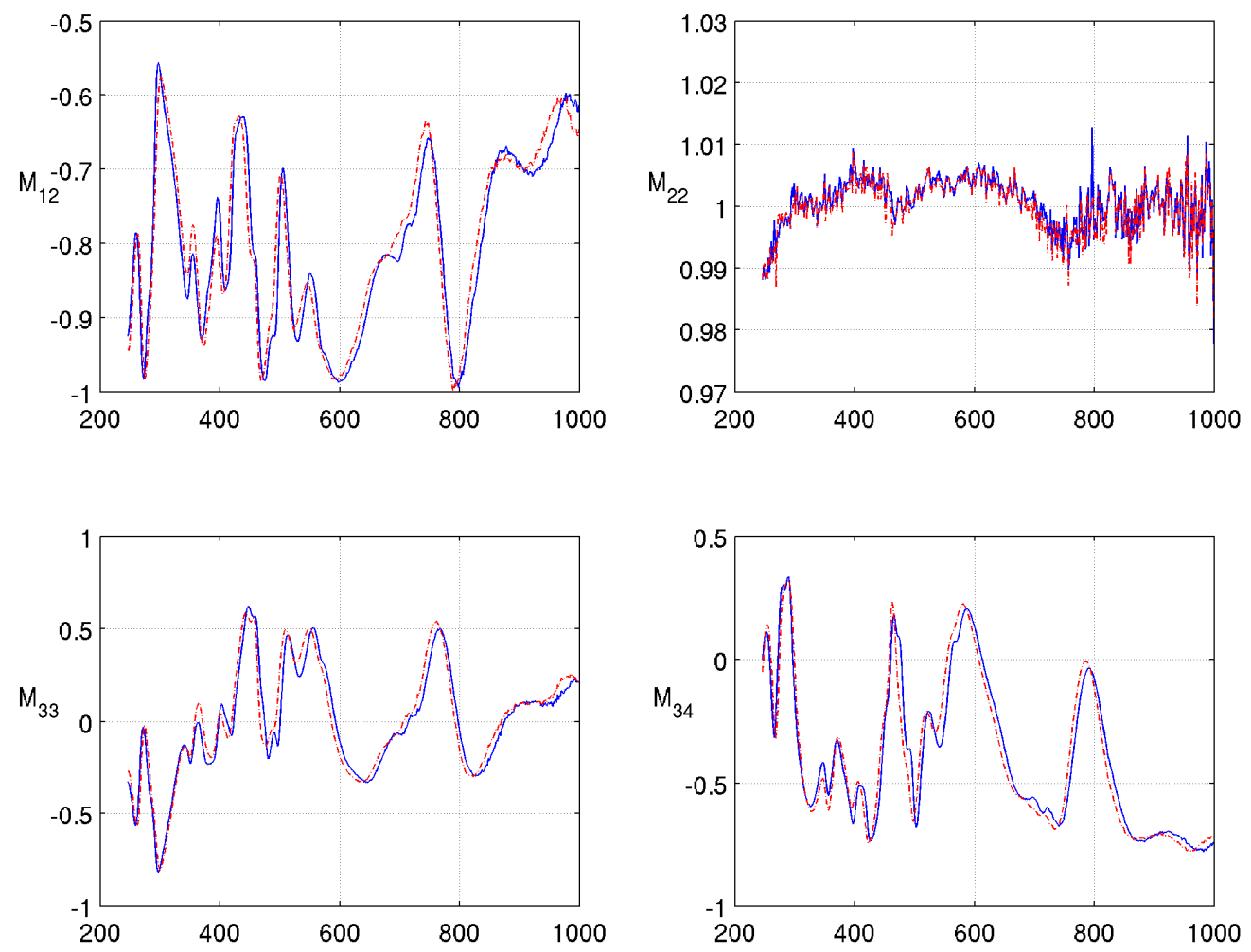

Figure 3. Spectral dependence (wavelength is in nanometers) of four non-zero elements of the Mueller matrix taken in the planar configuration for the reference grating (blue solid lines) and the artificial LER grating (red dash dotted lines).

For a comparison, we also include data measured at $90^{\circ}$ azimuth (light incident along the lines) as shown in Fig. 4. The situation is similar, as there are also no optical cross-polarization effects for symmetric profile line gratings at an azimuth of $90^{\circ}$. Nevertheless, we can see more interesting behavior of the element $M_{22}$, which deviates significantly from unity in some particular spectral regions. This effect is due to the finite NA, which was explained in the previous section, and it has significant impact on the measured data in spectral regions with very sharp features. The reason for the substantial differences between elements $M_{22}$ taken at azimuths of $0^{\circ}$ and $90^{\circ}$ is the typical behavior of the optical response of line gratings. Namely, while data vary rather slowly near azimuths close to $0^{\circ}$, they vary to a much greater degree near $90^{\circ}$. The finite NA is not only responsible for the changes due to the incidence angle averaging, but also due to the azimuthal angle averaging. As a result, depolarization effects will appear in the measured data to a greater extent near the azimuthal angle of $90^{\circ}$. 

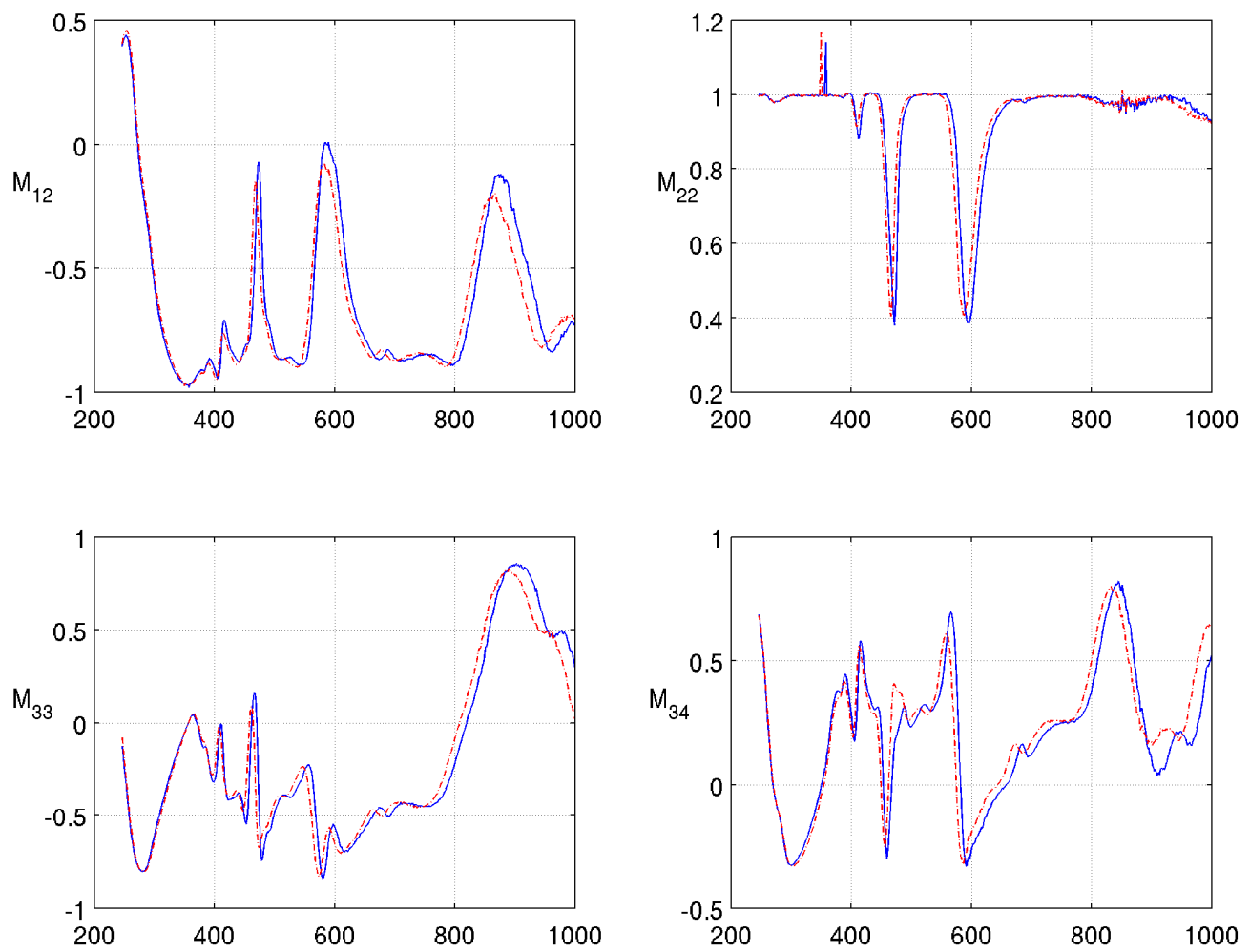

Figure 4. Spectral dependence (wavelength is in nanometers) of four non-zero elements of the Mueller matrix taken at $90^{\circ}$ azimuth for the reference grating (blue solid lines) and the artificial LER grating (red dash dotted lines).

The results of fitting the azimuthally dependent Mueller matrix data are shown in Fig. 5. Profiles acquired at azimuthal angles from $0^{\circ}$ to $90^{\circ}$ (with a step of $10^{\circ}$ ) are plotted together to illustrate the stability of the fits and the consistency in the profile shape. Figure 5 shows enormous stability of the middle line-width, which is usually the most precisely determined dimension using optical methods. In fact, changes of the middle line width values between different azimuths are in the sub-nanometer scale. It is possible to notice that the hardest parts of the profile to be determined (the most correlated values) correspond to the top and the bottom of the grating where, unlike for the middle line width, the differences are noticeable. The main purpose of showing Fig. 5 is to illustrate the stability of the parameter determination which can be achieved for all possible configurations, not only for one selected, i.e., planar, configuration. The advantage of using multiple azimuth configurations is that validity of the grating model can be tested; if it did not represent the true profile of the line, it is unlikely that the local minima of the fits would yield the same profile parameters. 


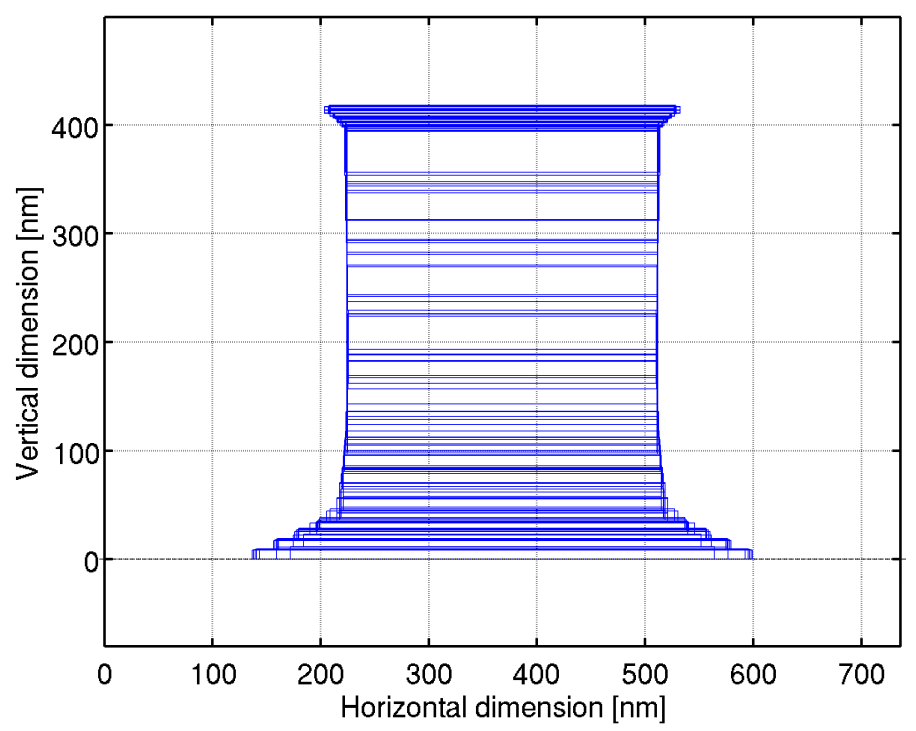

Figure 5. Simultaneous plot of profiles fitted at ten different azimuthal angles from $0^{\circ}$ to $90^{\circ}$.

The final results of our work are shown in Fig. 6. The figure represents RMSE values acquired using the same profile model for the reference and the perturbed gratings. We can see a consistent increase of RMSE values for the LER grating as compared to the reference grating. The increase is not that large as was observed in our previous work on periodically designed LWR [5], but it is still evident. The absolute values of RMSE are larger than for the case in Ref. [5] and also ratios between RMSE differences and RMSE of the reference target are less favorable. In order to increase the contrast between RMSE values acquired on the reference and the perturbed gratings, the further improvement of the model is required, which means more details need to be introduced into the profile parameterization, while avoiding unacceptable correlations between parameters. Since the correlations between parameters were rather favorable, we believe that further improvements are possible. We stress here that keeping consistency over all azimuthal configurations is important, as it helps us to avoid the pitfall of selecting a profile having smaller RMSE at certain azimuths but less stable over other azimuths.

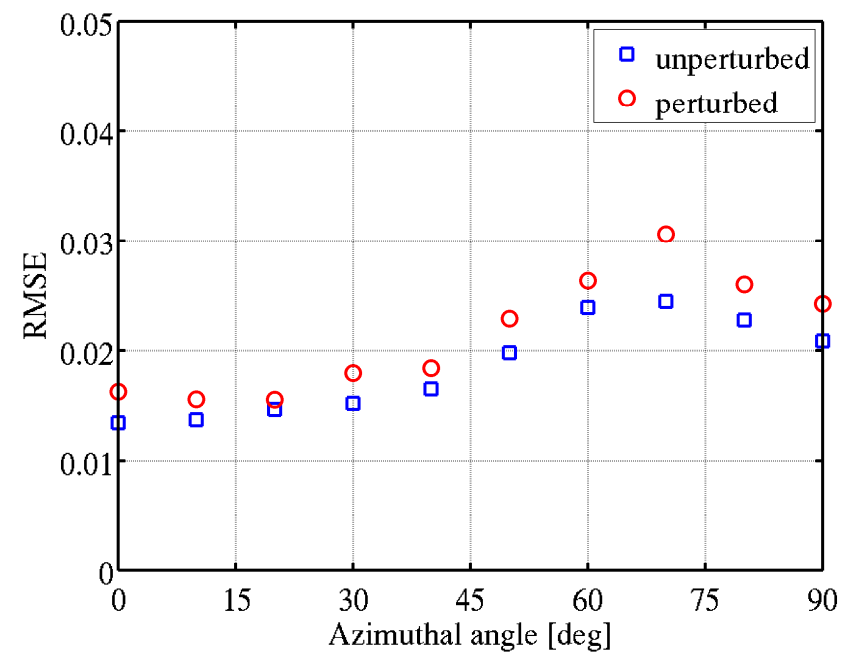

Figure 6. Azimuthal dependence of RMSE acquired by fitting reference (blue boxes) and artificially perturbed (red circles) line gratings. 


\section{CONCLUSIONS}

We have demonstrated a study of spectroscopic Mueller matrix ellipsometry to pseudo-random LER. In order to systematically evaluate the sensitivity of the method, we have manufactured a near perfect grating (that is, small natural LER) and artificially perturbed grating using a pseudo-random approach. The method involves using multiple azimuthal configurations in order to provide information on the stability of the results. The first step was to determine a fourtrapezoid-based profile model for the reference grating, which, when finite numerical aperture is correctly taken into account, corresponds well to the measured data and provide reasonable RMSE. In the next step, we applied the same model to the artificially perturbed grating in order to see differences in RMSE. Indeed, a consistent increase of RMSE for all azimuthal angles was observed confirming that we are sensitive to the manufactured LER. This conclusion is important in the context of the presence of significant NA which generally decreases the sensitivity to LER. We have also proposed that for further improvement of the sensitivity or the contrast between RMSE values acquired for the reference and the artificial LER gratings, a more detailed model of the profile can be considered.

\section{REFERENCES}

[1] Silver, R., Germer, T., Attota, R., Barnes, B.M., Bunday, B., Allgair, J., Marx, E. and Jun, J. "Fundamental limits of optical critical dimension metrology: a simulation study,” Proc. SPIE 6518, 65180U (2007).

[2] Germer, T.A. and Patrick, H.J., "Effect of bandwidth and numerical aperture in optical scatterometry,” Proc. SPIE 7638, 76381F (2010).

[3] Bergner, B.C., Germer, T.A. and Suleski, T.J., "Effect of line-width roughness on optical scatterometry measurements,” Proc. SPIE 7272, 72720 U (2009).

[4] Bergner, B.C., Germer, T.A. and Suleski, T.J., "Effective medium approximations for modeling optical reflectance from gratings with rough edges," J. Opt. Soc. Am. A 27, 1083-1090 (2010).

[5] Foldyna, M., Germer, T.A., Bergner, B.C. and Dixson, R.G., "Generalized ellipsometry of artificially designed line width roughness,” Thin Solid Films 519, 2633-2636 (2011).

[6] Moharam, M.G. and Gaylord, T.K., "Rigorous coupled-wave analysis of planar-grating diffraction,” J. Opt. Soc. Am. 7, 811-818 (1981).

[7] Moharam, M.G. and Gaylord, T.K., "Three-dimensional vector coupled-wave analysis of planar-grating diffraction," J. Opt. Soc. Am. 73, 1105-1112 (1983).

[8] Lalanne, P. and Morris, G.M., "Highly improved convergence of the coupled-wave method for TM polarization," J. Opt. Soc. Am. A 13, 779-784 (1996).

[9] Lifeng, L., "Use of Fourier series in the analysis of discontinuous periodic structures,” J. Opt. Soc. Am. A 13, 18701876 (1996).

[10]Lifeng, L., "Formulation and comparison of two recursive matrix algorithms for modeling layered diffraction gratings,” J. Opt. Soc. Am. A 13, 1024-1035 (1996).

[11] Rokushima, K. and Yamakita, J., “Analysis of anisotropic dielectric gratings,” J. Opt. Soc. Am. 73, 901-908 (1983).

[12] Jones, R.C., “A New Calculus for the Treatment of Optical Systems,” J. Opt. Soc. Am. 31, 488-493 (1941).

[13] Foldyna, M., De Martino, A., Ossikovski, R., Garcia-Caurel, E. and Licitra, C., "Characterization of grating structures by Mueller polarimetry in presence of strong depolarization due to finite spot size," Opt. Commun. 282, 735-741 (2009). 